\title{
Study outcome of continuous positive airway pressure in obstructive sleep Apnea
}

\author{
Krishna Potdukhe ${ }^{1 *}$, Rajesh Viswakarma², Kalpesh Patel ${ }^{3}$, Dipesh Darji ${ }^{4}$
}

${ }^{1}$ Senior Resident, ${ }^{3}$ Associate Professor, ${ }^{4}$ Assistant Professor, Department of Otorhinolaryngology, B J Medical College, Civil Hospital, Ahmedabad, Gujrat, INDIA.

2Professor and HOD Department of Otorhinolaryngology, Apollo Hospital, Ahmedabad, Gujrat, INDIA.

Email: krishnapotdukhe92@gmail.com

\begin{abstract}
Aim: The prevalence of Obstructive Sleep Apnea (OSA) in the Indian population is three-fold higher in men as compared to women. Obesity is believed to predispose to OSA because of mass loading of the upper airway. CPAP is the leading therapy for sleep apnea. To study and evaluate effectiveness of continuous positive airway pressure therapy in Obstructive Sleep Apnea. Material and Methods: This is a prospective cohort study, around 20 patients were diagnosed to have Obstructive Sleep Apnea and were given CPAP therapy, outcome of the treatment was measured by Apnea Hypopnea Index (AHI) at 6 months of period. Result: All patients had tremendous improvement in symptoms and clinical features. 13 out of 20 patients had $\mathrm{AHI}<5$ showing $65 \%$ success rate. Conclusion: CPAP is highly effective in controlling symptoms and improving quality of life.

Keywords: Obstructive Sleep Apnea, Continuous Positive Airway Pressure, Apnea Hypopnea Index.
\end{abstract}

*Address for Correspondence:

Dr. Krishna Potdukhe, Senior Resident, Department of Otorhinolaryngology, B J Medical College, Civil Hospital, Ahmedabad.380007

Email: krishnapotdukhe92@gmail.com

Received Date: 07/06/2019 Revised Date: 28/07/2019 Accepted Date: 20/08/2019

DOI: https://doi.org/10.26611/10161131

\begin{tabular}{|l|l|}
\hline \multicolumn{2}{|c|}{ Access this article online } \\
\hline Quick Response Code: & Website: \\
\hline & www.medpulse.in \\
& \\
\hline
\end{tabular}

\section{INTRODUCTION}

Obstructive Sleep Apnea (OSA) is the most common type of sleep Apnea and is caused by obstruction of the upper airway. According to American Academy of Sleep Medicine, Obstructive Sleep Apnea Syndrome (OSAS) is characterized by recurrent episodes of partial or complete upper airway obstructions during sleep. ${ }^{1}$ This manifest as a reduction (hypopnea) or complete cessation (Apnea) of airflow despite ongoing inspiratory efforts resulting in oxygen desaturations and arousals. With the recent advances in the understanding of the disease pathophysiology, various studies show that untreated OSA can lead to increased road traffic accident to high cardiovascular morbidity and even in severe case death may occur. Therefore diagnosis and management of OSA is very much important to improve the quality of life of the patient. The goal of OSA treatment is to alleviate airway obstruction during sleep. The standard first-line OSA treatment involves continuous positive airway pressure (CPAP) devices, which deliver compressed air into the airway to keep it open. Many patients do not tolerate CPAP and often do not adhere to the instructions for many reasons, including discomfort, skin irritation, noise, and claustrophobia. To improve adherence, many technological modifications have been made to CPAP devices (mostly in alterations of when air pressure is delivered), although the utility of the modified devices is unknown. Because adherence is often an issue in OSA treatment, additional patient education or interventions may be warranted. Various surgeries like tonsillectomy, adenoidectomy, UPPP, tongue channeling, turbinoplasty are also suggested. We present a study to evaluate the effectiveness of CPAP therapy in obstructive sleep Apnea. 


\section{MATERIALS AND METHOD}

This is a prospective cohort study, between May, 2016 to May, 2018 around 20 patients were diagnosed to have AHI $>5$ by Polysomnography and underwent CPAP therapy for OSA at Department of ENT and Head-Neck Surgery, B. J. Medical College and Civil Hospital, Ahmedabad. All the patients included in the study were explained about the purpose and use of the study and after their consent only they were included in the study. All the patients were evaluated in ENT OPD preoperatively for history, general examination and ENT examination. Overnight polysomnography was done in all suspected cases of OSA by pulmonologist. Patients who had AHI $>5$ were investigated for routine blood and radiological examination if indicated. All the patients were given CPAP therapy during period between May, 2016 to May, 2018 at Civil Hospital, Ahmedabad. All the study patients were subjected to a detailed history taking, examination, and investigations. To begin the CPAP titration, the patient was asked if they are a mouth breather or a nasal breather to ensure that an appropriate trial mask is chosen. The titration/trial mask is then fitted to the patient and they are asked to practice putting it on their face, preferably in front of a mirror. Once the patient is comfortable handling the mask, it is connected to the titration PAP machine, and the starting procedure is demonstrated. The patient should spend a few minutes with the PAP machine on, to get an idea of how the pressure will feel. In CPAP machine beginning at very low pressures, typically $4-6 \mathrm{cmH} 2 \mathrm{O}$, increasing until it reaches a therapeutic level. During the 3-7 day trial, follow-up should be continuously maintained. Patients were followed at 15 days, 1 month and 6 months. Outcome of the treatment was measured by AHI at 6 months of period. At the end of study all the data was compiled, reviewed and ultimately results obtained.

\section{OBSERVATION AND RESULT}

In the present study 20 patients were taken for CPAP therapy. The cases were observed in prospective manner including their age, sex, presenting complains, clinical features, examination, pre-therapy and post-therapy polysomnography (AHI) findings and complications. Table 1 shows distribution of the patient according to their age and sex. In this study $55 \%$ of the patients were between 40 to 60 years of age and $75 \%$ are male.

Table 1: Age and Sex distribution of cases

\begin{tabular}{cccc}
\multicolumn{4}{c}{ Table 1: Age and Sex distribution of cases } \\
\cline { 2 - 5 } Age group(years) & No of patients & Male & Female \\
\hline $20-40$ & $4(20 \%)$ & 4 & 0 \\
$40-60$ & $11(55 \%)$ & 8 & 3 \\
$60-80$ & $5(25 \%)$ & 3 & 2 \\
\hline Total & 20 & 15 & 5 \\
\hline
\end{tabular}

Table 2 shows most common clinical finding in this study was excessive daytime sleepiness which was present in $80 \%$ cases. Next most common finding was fatigue and non- refreshing sleep which was present in almost $60 \%$ cases.

\begin{tabular}{ccc}
\multicolumn{3}{c}{ Table 2: Distribution if clinical features } \\
\hline Clinical features & No. Patients (out of 20) & Percentage (\%) \\
\hline Excessive Daytime sleeping (EDS) & 16 & 80 \\
Fatigue & 13 & 65 \\
Non refreshing sleep & 12 & 60 \\
Breath holds & 5 & 25 \\
Increased total sleep duration & 3 & 15 \\
Mood changes & 2 & 10 \\
Nocturia & 1 & 5 \\
\hline
\end{tabular}

In table 3 out of 20, 5 patients had Epworth score below 10. Though 16 out of 20 patients gave history of excessive daytime sleeping 15 patients gave above 10 score.

TABLE 3: Epworth Sleeping scale in patients having excessive daytime sleeping

\begin{tabular}{ccc}
\hline Epworth Sleeping scale & Pre op No. Patients (out of 20) & Post op No. Patients (out of 20) \\
\hline $0-10$ & 5 & 18 \\
$11-24$ & 15 & 2 \\
\hline
\end{tabular}

11 patients had significant positive past history. 8 patients were having hypertension and all were on treatment. 3 patients were known case of hypothyroidism out of which 2 patients were newly diagnosed. 5 patients had a habit of smoking. 6 patients had alcohol habit. 
Table 4: Apnea-hypopnea index (AHI)

\begin{tabular}{ccc}
\hline AHI & Pre op No. Patients (out of 20) & Post op No. Patients (out of 20) \\
\hline <5 No OSA & 0 & 13 \\
5-14 Mild OSA & 14 & 4 \\
15-29 Moderate OSA & 5 & 3 \\
$\geq 30$ Severe OSA & 1 & 0 \\
\hline
\end{tabular}

Table 4 shows, 13 patients out of 20 patients had AHI $<5$. Around $65 \%$ success rate was seen. 4 patients complain of nasal dryness, congestion and nasal abrasion. These adverse effects in patients treated with CPAP could be alleviated with termination or modification of the treatment.

\section{DISCUSSION}

Obstructive sleep apnea (OSA) is increasingly being recognized as an important health issue in the last two to three decades. There is accumulating evidence that OSA is being considered as an independent risk factor for hypertension, glucose intolerance/ diabetes mellitus, cardiovascular diseases and stroke, leading to increased cardio metabolic morbidity and mortality. With increasing awareness of this disease entity and associated complications in our society, there have been increased referrals to sleep physicians and surgeons or expertise for further investigations and diagnostic evaluation. Early recognition and treatment of obstructive sleep apnea may prevent from adverse health consequences. ${ }^{2}$ OSA as a disease is just a tip of iceberg what we can see. Despite the recent advances in diagnostic technology in the field of sleep medicine and increased awareness of OSA in the public, a majority of those affected are still undiagnosed. ${ }^{3}$ Therefore, it is important for us to be competent to recognize and identify those affected subjects for early and appropriate treatments. This study includes the early identification of OSA cases and effective treatment. Surgical management of OSA is indicated when a surgically correctable abnormality is believed to be the source of the problem and the patient has tried continuous positive airway pressure (CPAP) without success. OSA can occur in any age group, but prevalence increases between middle and older age group. Mechanisms proposed for the increased prevalence of sleep apnoea in the elderly include increased deposition of fat in the parapharyngeal area, lengthening of the soft palate, and changes in body structures surrounding the pharyn $x^{4}$. In this study $55 \%$ of the patients were between 40 to 60 years of age. Data from the community-based sleep heart health study have shown that disease prevalence increases steadily with age and reaches a plateau after the age of 60 years. Clinical studies have shown that, the ratio of men to women is in the range from 5 to $8: 1 .^{5}$ Epidemiologic studies have confirmed the higher prevalence of obstructive sleep apnea in men but report a lower maleto-female ratio in the range 2 to $3: 1 .^{6,7,8,9}$ In this study
$21(70 \%)$ patients were male and female patients were 9 $(30 \%)$. The clinical manifestations of OSAS are related to obstruction of the upper airway, fragmented sleep, and the respiratory and cardiovascular consequences of disordered breathing. Excessive daytime somnolence is a key feature of OSAS resulting from disrupted sleep. Patients may report that they frequently fall asleep during the day while driving, working, reading and watching television ${ }^{10}$. Chronic daytime sleepiness also leads to poor work performance and decreased productivity. In this study most common clinical feature in this study was excessive daytime sleeping which was present in $70 \%$ cases. The Epworth Sleepiness Scale (ESS) is an effective instrument used to measure average daytime sleepiness. The ESS differentiates between average sleepiness and excessive daytime sleepiness that requires intervention. The patient self-rates on how likely it is that he/she would doze in eight different situations. Though ESS is not an appropriate tool for measuring changes in sleep over a period of hours, the ESS may be used for both initial assessment and ongoing comparative measurements with older adults across the health care continuum. Numerous studies using the ESS have supported high validity and reliability. The ESS is a subjective measure of sleepiness. Treatment options for OSA include weight loss, dental devices or oral appliance therapy, surgical procedures, and CPAP. CPAP is the mainstay of medical treatment for OSA and involves the use of pump to deliver air into the nose or mouth via a mask during sleep. Positive pressure is generated by airflow, which opens up the airway and prevents the soft tissue from collapsing. The effectiveness of CPAP is often limited by poor adherence rates underscoring the importance of identifying barriers to adherence and developing interventions to improve adherence.

Studies evaluating the effect of CPAP on medical cost are limited and findings are not consistent.

\section{CONCLUSION}

OSA usually affects middle and older aged men and we can call it a disease of upper class as almost always it is associated with the obesity. India is a country with high prevalence of smokers, alcoholics and tobacco 
chewers which further increases chances to have an obstructive sleep apnea to that person. Diagnosis of OSA is usually done by presence of its typical features and polysomnography (PSG) which is considered gold standard in diagnosis of OSA. Apnea-Hypopnea index (AHI) is an important marker of the severity of the disease. Treatment of OSA starts from life style modifications such as weight reduction, cessation of smoking and alcohol and control of existing comorbidities. In our study, we conclude that CPAP is highly effective in controlling symptoms, improving quality of life and reducing the clinical consequences of sleep apnea but compliance is low due to its side effects.

\section{REFERENCES}

1. Sullivan CE, Issa FG, Berthon-Jones M, Eves L. Reversal of obstructive sleep apnoea by continuous positive airway pressure applied through the nares. Lancet 1981;1:862-5.

2. Indian J Med Res 131, February 2010, pp 165-170. Obstructive sleep apnoea: Definitions, epidemiology and natural history Jamie C.M. Lam, S.K. Sharma* and Bing Lam.

3. Pagel JF. The burden of obstructive sleep apnoea and associated excessive sleepiness. J Fam Pract 2008; 57 (8 suppl): S3-8.
4. Eikermann M, Jordan AS, Chamberlin NL, G autam S, Wellman A, Lo YL, et al The influence of aging on pharyngeal collapsibility during sleep. Chest 2007;131:1702-9.

5. Strohl KP, Redline S. Recognition of obstructive sleep apnea. Am J Respir Crit Care Med 1996;154:279-289.

6. Young T, Shahar E, Nieto FJ, Redline S, Newman AB, Gottlieb DJ, Walsleben JA, Finn L, Enright P, Samet JM. Predictors of sleep-disordered breathing in community-dwelling adults: the Sleep Heart Health Study. Arch Intern Med 2002;162:893-900.

7. Duran J, Esnaola S, Rubio R, Iztueta A. Obstructive sleep apnea-hypopnea and related clinical features in a population-based sample of subjects aged 30 to 70 yr. Am J Respir Crit Care Med 2001;163:685-689.

8. Bixler EO, Vgontzas AN, Lin HM, Ten Have T, Rein J, Vela-Bueno A, Kales A. Prevalence of sleepdisordered breathing in women: effects of gender. Am J Respir Crit Care Med 2001;163:608-613.

9. Redline S, Kump K, Tishler PV, Browner I, Ferrette $\mathrm{V}$. Gender differences in sleep disordered breathing in a community-based sample. Am J Respir Crit Care Med 1994;149:722-726.

10. Bahammam A, Kryger M. Decision making in obstructive sleepdisordered breathing: putting it all together. Otolaryngol Clin North Am 1999; 32(2):33348 .

\section{Source of Support: None Declared Conflict of Interest: None Declared}

